\title{
Does prior administration of rtPA influence acute ischemic stroke clot composition? Findings from the analysis of clots retrieved with mechanical thrombectomy from the RESTORE registry
}

\author{
Rosanna Rossi ${ }^{1,2} \cdot$ Sara Molina ${ }^{1,2} \cdot$ Oana Madalina Mereuta ${ }^{1,2} \cdot$ Andrew Douglas $^{1,2} \cdot$ Seán Fitzgerald ${ }^{1}$. \\ Ciara Tierney ${ }^{1,2}$ - Abhay Pandit ${ }^{2}$. Paul Brennan ${ }^{3}$. Sarah Power ${ }^{3}$. Alan O'Hare ${ }^{3}$ - Michael Gilvarry ${ }^{4}$. Ray McCarthy ${ }^{4}$. \\ Georgios Magoufis ${ }^{5}$. Georgios Tsivgoulis ${ }^{6} \cdot$ András Nagy $^{7} \cdot$ Ágnes Vadász $^{7} \cdot$ Katarina Jood $^{8,10} \cdot$ Petra Redfors $^{8,10}$. \\ Annika Nordanstig ${ }^{8,10} \cdot$ Erik Ceder $^{9} \cdot$ Dennis Dunker $^{9} \cdot$ Jeanette Carlqvist ${ }^{9} \cdot$ Klearchos Psychogios $^{5} \cdot$ István Szikora $^{7}$. \\ Turgut Tatlisumak $^{8,10} \cdot$ Alexandros Rentzos $^{9} \cdot$ John Thornton $^{3} \cdot$ Karen M. Doyle ${ }^{1,2}$ (1)
}

Received: 22 May 2021 / Revised: 29 June 2021 / Accepted: 15 August 2021 / Published online: 20 August 2021

(c) The Author(s) 2021

\begin{abstract}
Background and purpose There is still much debate whether bridging-therapy [intravenous thrombolysis (IVT) prior to mechanical thrombectomy (MT)] might be beneficial compared to MT alone. We investigated the effect of IVT on size and histological composition of the clots retrieved from patients undergoing bridging-therapy or MT alone.

Methods We collected mechanically extracted thrombi from 1000 acute ischemic stroke (AIS) patients included in RESTORE registry. Patients were grouped according to the administration (or not) of IVT before thrombectomy. Gross photos of each clot were taken and Extracted Clot Area (ECA) was measured using ImageJ software. Martius Scarlett Blue stain was used to characterize the main histological clot components [red blood cells (RBCs), fibrin (FIB), platelets/other (PTL)] and Orbit Image Analysis was used for quantification. Additionally, we calculated the area of each main component by multiplying the component percent by ECA. Chi-squared and Kruskal-Wallis tests were used for statistical analysis.

Results 451 patients (45\%) were treated with bridging-therapy while $549(55 \%)$ underwent MT alone. When considering only percent histological composition, we did not find any difference in RBC\% $(P=0.895)$, FIB\% $(P=0.458)$ and PTL\% $(P=0.905)$. However, bridging-therapy clots were significantly smaller than MT-alone clots [32.7 (14.8-64.9) versus 36.8 $\left.(20.1-79.8) \mathrm{mm}^{2}, N=1000, \mathrm{H1}=7.679, P=0.006 *\right]$. A further analysis expressing components per clot area showed that clots retrieved from bridging-therapy cases contained less RBCs $\left[13.25(4.29-32.06)\right.$ versus $14.97(4.93-39.80) \mathrm{mm}^{2}, \mathrm{H} 1=3.637$, $P=0.056]$ and significantly less fibrin $\left[9.10(4.62-17.98)\right.$ versus $\left.10.54(5.57-22.48) \mathrm{mm}^{2}, \mathrm{H} 1=7.920, P=0.005^{*}\right]$ and platelets/other [5.04 (2.26-11.32) versus $\left.6.54(2.94-13.79) \mathrm{mm}^{2}, \mathrm{H} 1=9.380, P=0.002 *\right]$ than MT-alone clots.

Conclusions Our results suggest that previous IVT administration significantly reduces thrombus size, proportionally releasing all the main histological components.
\end{abstract}

Keywords Stroke $\cdot$ Mechanical thrombectomy $\cdot$ Bridging-therapy $\cdot$ Thrombus histology $\cdot$ Thrombus size

\section{Introduction}

Current evidence-based treatments for acute ischemic stroke (AIS) in Large Vessel Occlusion (LVO) include both intravenous thrombolysis (IVT) and mechanical thrombectomy (MT) [1-4]. Current guidelines recommend proceeding with

Karen M. Doyle

karen.doyle@nuigalway.ie

Extended author information available on the last page of the article both treatments as soon as possible. Patients may be unsuitable for IVT but still suitable for MT. While the effectiveness of MT compared to IVT alone has been extensively demonstrated [5-10], conflicting results have been reported when comparing bridging-therapy to MT thrombectomy alone [11-15]. Questions remain as to whether pre-treatment with intravenous thrombolytics significantly affects MT success and patient outcome. There are several ongoing randomized controlled clinical trials addressing this issue.

Investigation of the properties of extracted thrombi can also help in this debate, since thrombus properties like 
size and composition could be influenced by IVT and have clinical significance. In this regard, we recently reported that clots extracted from patients undergoing bridgingtherapy are significantly smaller compared to those of patients undergoing MT alone, although there was no difference in final recanalization outcome [16]. Furthermore, several studies have reported the wide heterogeneity in thrombus composition [17-19], which is likely to be influenced by several factors, including etiology and perhaps also thrombolysis [20].

In this study, we investigated the effect of previous IVT administration in clots removed by MT on histological composition of thrombi retrieved from a larger cohort of 1000 AIS patients, both in terms of percentage composition and also as a function of clot size.

\section{Materials and methods}

\section{Patient cohort}

We collected clots from 1000 AIS patients as part of the RESTORE registry of acute ischemic stroke clots. The RESTORE Registry is a registry of thrombotic material extracted via mechanical thrombectomy from patients suffering from AIS and it accounts for clots collected during the period February 2018 to December 2019 from four stroke centres in Europe. A main aim of the Registry is to analyse clots from AIS patients and correlate clot characteristics with clinical and procedural information. For this reason, patients for which no clot could be extracted are not part of the Registry and we have no information about these patients. This multi-centre prospective study was conducted in accordance with the ethical standards of the Declaration of Helsinki and its amendments [21], by approval of the regional hospital ethics committees and National University of Ireland Galway research ethics committees (16-SEPT-08).

We included only patients $>18$ years, having been treated with mechanical thrombectomy for AIS and whose thrombus material was available for analysis. For each patient an anonymized data abstraction form was collected, which contained pertinent procedural data, including if IVT was administered, occlusion location, type of device used for MT, number of passes for clot removal, suspected etiology and final modified Treatment In Cerebral Ischemia (mTICI) score [22]. Grade of reperfusion after endovascular procedure was assessed by anteroposterior/lateral Digital Subtraction Angiography and certified by at least two radiologists at the hospital site. Suspected stroke etiology was reported according to the TOAST classification system [23].
Measurement of extracted thrombus size: Extracted Clot Area

After the endovascular treatment, extracted thrombi were collected per pass and shipped in $10 \%$ formalin to NUI Galway, where a gross photo of each was taken with a Canon EOS 1300D Camera. Extracted Clot Area (ECA) was used as an estimate of the size of extracted clot and was measured as previously reported [17, 24, 25]. In brief, to measure the area of a fragment, the gross photo was opened with ImageJ software, the scale was set and the Polygon tool was used to draw a region of interest around the fragment. For passes involving multiple fragments we measured and summed the area of each fragment to obtain the ECA of each pass. Then, for cases involving multiple passes we summed the ECA of each pass to obtain the overall ECA.

\section{Histological component analysis}

After having been photographed, thrombi were placed in histological cassettes for tissue processing and were paraffin embedded. $3 \mu \mathrm{m}$ sections were cut with a microtome. Martius Scarlett Blue (MSB) staining was performed to identify the standard clot tissue components: red blood cells (RBCs), white blood cells (WBCs), fibrin (FIB), platelets/other (PTL) and non-typical thrombus components such as collagen and calcification [26]. We used machine-learning Orbit image analysis (www.Orbit.bio) for quantification purposes $[27,28]$. Briefly, we created exclusion and inclusion models to distinguish regions to be excluded (e.g. background and artefact) and regions containing the tissue components of interest, enabling quantitative assessment of the proportion of each component within each clot.

In addition, we calculated the area occupied by each main component (RBC, FIB, PTL) by multiplying the component percent by the relevant ECA. For cases involving multiple passes, we summed the values of ECAxComponent for all passes.

\section{Statistical analysis}

IBM SPSS-25 software was used for statistical analysis. Kolmogorov-Smirnov test and Shapiro-Wilk test indicated that quantitative variables did not follow a standard normal distribution. Therefore, the non-parametric chi-squared and Kruskal-Wallis tests were used to assess statistically significant difference among the groups, with a level of statistical significance set at $p<0.05$ (two-sided). Results are reported as median [IQ1-IQ3] or number and \% of cases. 


\section{Results}

\section{Baseline characteristics of the patients}

Among the 1000 cases considered, 451 patients (45\%) were treated with bridging-therapy while $549(55 \%)$ were treated with mechanical thrombectomy alone. The total number of passes performed was 2365 , with at least some clot material extracted in 1496 passes (63\%), while 869 attempts were performed with no clot extraction (37\%). Baseline clinical characteristics of both groups of patients are reported in Table 1. There was no significant difference between the two populations in terms of suspected etiology $\left(X^{2}=3.771\right.$, $P=0.438)$, first-line approach used $\left(X^{2}=2.417, P=0.120\right)$ or revascularisation outcome $(\mathrm{H} 1=1.189, P=0.275)$; Table 1.

\section{Occluded vessel location}

Occluded vessel location was defined by an expert radiologist at the hospital site at the beginning of procedure. In the majority of the cases, $(881,88 \%)$ the ischemic territory was located in the anterior circulation. In 100 cases (10\%), the occlusion was located in the posterior circulation, while in 17 cases $(2 \%)$ both anterior and posterior territories were involved. Occlusion involved a single vessel in $78 \%$ of cases (782 single occlusions), while in 216 cases (22\%), two or more vessels were occluded at the same time. Specific vessels occluded, for the overall population, and according previous IVT administration, are reported in Table 2 . There was no significant difference in occlusion type observed between the bridging-therapy and mechanical thrombectomy-alone groups (anterior/posterior occlusion: $X^{2}=4.575, P=0.102$; singular/multiple occlusion: $X^{2}=0.061, P=0.804$ ).

\section{Analysis of Extracted Clot Area}

Analysis of size of extracted clot (expressed as Extracted Clot Area) showed that clots from patients undergoing bridging-therapy were statistically significantly smaller than clots retrieved from patients treated with MT alone, (Fig. 1a; median ECA was, respectively, 32.7 [14.8-64.9] versus 36.8 $\left.[20.1-79.8] \mathrm{mm}^{2} N=1000, \mathrm{H} 1=7.679, P=0.006^{*}\right)$.

Table 1 Baseline clinical characteristics of the overall cohort of patients and divided by the two groups of patients, bridging-therapy and mechanical thrombectomy alone

\begin{tabular}{|c|c|c|c|}
\hline Suspected etiology* & $\begin{array}{l}\text { Overall cohort of } \\
\text { patients }(N=1000)\end{array}$ & $\begin{array}{l}\text { Bridging-therapy } \\
\text { cases }(N=451)\end{array}$ & $\begin{array}{l}\text { Mechanical thrombec- } \\
\text { tomy-alone cases } \\
(N=549)\end{array}$ \\
\hline Patients with cardioembolic suspected etiology & $346(34.6 \%)$ & $152(33.7 \%)$ & $194(35.3 \%)$ \\
\hline Patients with large artery atherosclerosis suspected etiology & $221(22.1 \%)$ & $111(24.6 \%)$ & $110(20.0 \%)$ \\
\hline Patients with other suspected etiology $\mathrm{y}^{\mathrm{a}}$ & $55(5.5 \%)$ & $22(4.9 \%)$ & $33(6.0 \%)$ \\
\hline Patients with cryptogenic etiology & $255(25.5 \%)$ & $115(25.4 \%)$ & $140(25.5 \%)$ \\
\hline \multicolumn{4}{|l|}{ First line approach ${ }^{\mathrm{b}}$} \\
\hline Aspiration & $657(65.7 \%)$ & $285(63.2 \%)$ & $372(67.8 \%)$ \\
\hline Stentriever & $342(34.2 \%)$ & $166(36.8 \%)$ & $176(32.1 \%)$ \\
\hline $\begin{array}{l}\text { Median number of attempts performed during the endovascular } \\
\text { procedure }\end{array}$ & $2(1-3)$ & $2(1-3)$ & $2(1-3)$ \\
\hline \multicolumn{4}{|l|}{ Final mTICI score ${ }^{c}$} \\
\hline mTICI 0 & $17(1.7 \%)$ & $6(1.3 \%)$ & $11(2.0 \%)$ \\
\hline mTICI 1 & $12(1.2 \%)$ & $6(1.3 \%)$ & $6(1.1 \%)$ \\
\hline mTICI $2 \mathrm{a}$ & $62(6.3 \%)$ & $30(6.7 \%)$ & $32(6.0 \%)$ \\
\hline mTICI 2b & $257(26.1 \%)$ & $106(23.7 \%)$ & $151(28.1 \%)$ \\
\hline mTICI $2 \mathrm{c}$ & $207(21.0 \%)$ & 97 (21.7) & $110(20.5 \%)$ \\
\hline mTICI 3 & $429(43.6 \%)$ & $202(45.2 \%)$ & $227(42.3 \%)$ \\
\hline
\end{tabular}

Data given as $N(\%)$ of cases or median (IQ1, IQ3)

${ }^{a}$ Other suspected etiology included: arterial dissection, pulmonary embolism, hypercoagulable states, or hematologic disorders

${ }^{\mathrm{b}}$ In 1 case a gooseneck snare has been used as first-line approach to remove a calcified clot

${ }^{\mathrm{c}}$ In 16 cases involving posterior circulation occlusion, it was not possible to assess final recanalization outcome (4 cases for bridging-therapy and 12 for MT alone)

*Suspected etiology was not recorded for 123 patients (12.3\%), 51 of which were treated with bridging-therapy (11.3\%) and 72 with MT alone $(13.11 \%)$ 
Table 2 Occluded vessels in the whole cohort of patients and in the two groups, bridging-therapy and mechanical thrombectomy alone

\begin{tabular}{llccc}
\hline Occlusion type & Occluded vessel(s) & All the cases $(N=1000)$ & $\begin{array}{l}\text { Bridging-therapy } \\
\text { cases }(N=451)\end{array}$ & $\begin{array}{l}\text { Mechanical thrombec- } \\
\text { tomy-alone cases } \\
(N=549)\end{array}$ \\
& & & $222(40.4 \%)$ \\
Singular occlusion $(N=781)$ & MCA,M1 & $424(42.4 \%)$ & $202(44.9 \%)$ & $71(13.0 \%)$ \\
& MCA,M2 & $124(12.5 \%)$ & $53(11.8 \%)$ & $1(0.2 \%)$ \\
& MCA,M3 & $5(0.5 \%)$ & $4(0.9 \%)$ & $2(0.4 \%)$ \\
& MCA not specified & $10(1.0 \%)$ & $8(1.8 \%)$ & $52(9.5 \%)$ \\
& Vertebro/basilar & $78(7.8 \%)$ & $26(5.8 \%)$ & $76(13.9 \%)$ \\
& ICA and ICA terminus & $131(13.1 \%)$ & $55(12.2 \%)$ & $6(1.1 \%)$ \\
& PCA & $8(0.8 \%)$ & $1(0.4 \%)$ & $5.2 \%)$ \\
& ACA & $2(0.2 \%)$ & $52(11.6 \%)$ & $57(10.4 \%)$ \\
& Tandem occlusions & $109(11 \%)$ & $19(4.2 \%)$ & $22(4.0 \%)$ \\
& Other dual occlusions & $41(4.1 \%)$ & $12(2.7 \%)$ & $15(2.7 \%)$ \\
& MCA, multiple segments/branches & $27(2.7 \%)$ & $16(3.6 \%)$ & $23(4.2 \%)$ \\
\hline
\end{tabular}

Data given as $N(\%)$ of cases

$M C A$ middle cerebral artery, ICA internal carotid artery, $P C A$ posterior cerebral artery, $A C A$ anterior cerebral artery, $C C A$ common carotid artery
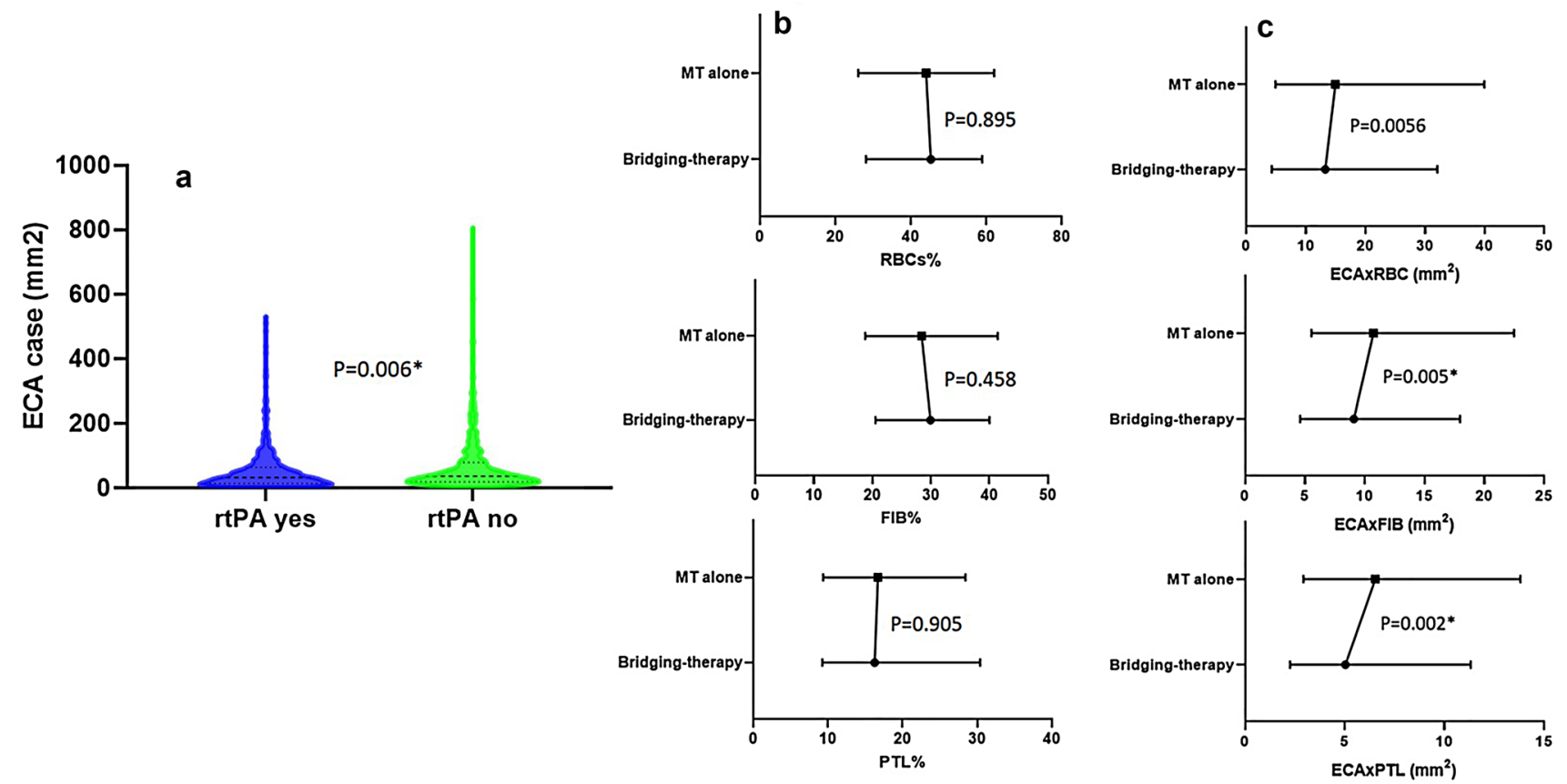

Fig. 1 Violin plot depicting variation and difference in Extracted Clot Area (a); Median values with IQ[1-3] range depicting difference in percentage of main histological components (b) and the area occu- pied by each main component, (expressed as ECA multiplied by component fraction) (c), between clots retrieved from patients undergoing bridging-therapy or mechanical thrombectomy alone
Bridging-therapy clots were also associated with a significantly lower number of fragments compared to MT alone (2 [1-4] versus 3 [2-5], $N=1000, \mathrm{H} 1=4.058$, $\left.P=0.044^{*}\right)$.

\section{Histological components analysis}

Histological component analysis revealed no difference in main histological components between bridging-therapy and 
MT-only clots (Fig. 1b). Median RBCs composition was 45 [28-59]\% for bridging-therapy and 44 [26-62]\% for MTalone clots $(\mathrm{H} 1=0.018, P=0.895)$; median FIB composition was 30 [21-40]\% for bridging-therapy and 29 [19-41]\% for MT alone $(\mathrm{H} 1=0.552, P=0.458)$; median PTL composition was 16 [9-30]\% for bridging-therapy and 17 [9-28]\% for MT alone $(\mathrm{H} 1=0.014, P=0.905)$.

Further analysis was then performed combining size and histological composition (Fig. 1c). The amount of RBC was lower in bridging-therapy compared to MT-only clots, although not statistically significant (median ECAxRBC was $13.25[4.29-32.06] \mathrm{mm}^{2}$ versus 14.97 [4.93-39.80] $\mathrm{mm}^{2}$, $\mathrm{H} 1=3.637, P=0.056$ ). The amount of FIB and PTL was statistically significantly lower in bridging-therapy clots compared to MT-only clots (respectively, median ECA $\times$ FIB was 9.10 [4.62-17.98] $\mathrm{mm}^{2}$ versus 10.54 [5.57-22.48] $\mathrm{mm}^{2}, \mathrm{H} 1=7.920, P=0.005^{*}$ and median ECA $\times$ PTL was $5.04[2.26-11.32] \mathrm{mm}^{2}$ versus 6.54 [2.94-13.79] $\mathrm{mm}^{2}$, $\left.\mathrm{H} 1=9.380, P=0.002^{*}\right)$, Fig. $1 \mathrm{c}$.

\section{Discussion}

There are several studies in the literature comparing final recanalization rate and other clinical outcomes for patients treated with bridging-therapy or MT alone, although there is not yet a clear consensus of opinion [11-16]. Nevertheless, bridging-therapy, i.e. the combination of IVT and mechanical thrombectomy, is currently the standard of care for large vessel occlusion (LVO) patients with a NIHSS-score of 6 or greater [29] provided that they fulfil tPA eligibility criteria [1-4]. Although it could be very informative, there is very little published about the influence of IVT on the characteristics of the retrieved clot, such as its general composition and structural organization. Our recent study comparing the size of extracted thrombi showed that bridging-therapy clots were significantly smaller than MT-only clots [16], a finding that is reproduced in the present larger study.

In the present study, we sought to assess if thrombolysis also altered clot composition. In this respect, we investigated percentage composition of main components and we also undertook a combined size and compositional analysis. Red blood cells were the main histological component for both groups of clots, followed by fibrin and then by platelets/other. We did not find any difference between the clots retrieved from the two groups of patients in terms of percent composition of main histological components. However, when size was taken into account, bridging-therapy clots contained less RBC $\left(\mathrm{mm}^{2}\right)$ and statistically significantly less fibrin and platelets/other $\left(\mathrm{mm}^{2}\right)$ compared to clots from patients treated with MT alone, directly relatable to the smaller size of bridging-therapy clots. Whilst the effect on RBC $\left(\mathrm{mm}^{2}\right)$ did not quite reach statistical significance, the very clear trend suggests that tPA administration reduces the content of all main components proportionally.

A previous study observed that previous IVT administration was associated with prominent changes in the architecture of the fibrin components and platelet count [30] of the retrieved clots. In particular, the phenomenon was termed as "thinning", referring to the dissociation of superficial layers of fibrin fibres, which was remarkably notable only in clots retrieved from patients treated with bridging-therapy [30]. An earlier study observed how clots retrieved after IVT treatment were more porous and with more branching points suggesting that thrombolytic therapy is associated with significant changes in the structural composition, and in particular in the fibrin network architecture of the retrieved clot [31]. Also a recent study investigating 3D organization of AIS thrombi showed how fibrin formed a grid-like or a sponge-like pattern in clots from patients pre-treated with IVT, with thinner and less densely packed fibrin fibres [32].

However, it has been also observed that sensitivity to fibrinolytic agents might depend on original thrombus composition. In this respect, RBC-rich thrombi seem to have higher sensitivity to rtPA [33, 34] probably due to their looser architecture [35], which allows for an increased penetration of thrombolytic agents into the clot [36].

Our findings show that IVT administration, even when it does not result in the complete dissolution of the occlusive thrombi, will reduce clot size. Our findings also suggest that fibrinolytic activity of IVT causes a proportional decrease of the three main histological clot components, maintaining the percentage ratio of main components within the clot. However, we acknowledge that we have no information of clot composition before mechanical thrombectomy, which would be needed for confirmation. Our findings also showed that bridging-therapy resulted in a lower number of fragments retrieved than with mechanical thrombectomy alone. However, further study is needed before any definitive conclusion can be drawn, as many factors during thrombectomy and during clot processing could impact on the number of fragments.

\section{Study limitations and strengths}

It must be acknowledged that our study on the effect of tPA is limited to cases where mechanical thrombectomy was performed and at least part of the clot was extracted. We do not have information for patients that recanalised after IVT alone or for those that had unsuccessful thrombectomy, with no clot extracted. The two patient cohorts differ clinically regarding eligibility for tPA. MT-only patients would have been ruled ineligible for tPA treatment for reasons such as time window exclusion, risk of haemorrhage or ongoing anticoagulation for atrial fibrillation [1-4]. Also, length of time since stroke onset could influence 
thrombus size. Patients receiving bridging-therapy often have a shorter time window from symptoms onset to hospital admission. Further studies should address the effect of time frame on thrombus size. Furthermore, we also acknowledge the lack of complete information about eventual anticoagulation regimes of the patient, dosage and specific thrombolytic agents administered to bridging-therapy patients, although this should be addressed in future studies.

However, despite these limitations, we observed a good similarity between the two groups of patients in terms of baseline and procedural characteristics including suspected etiology, occluded vessel treated, first-line approach for endovascular procedure and final revascularisation outcome. Furthermore, the main strength of this study is the large patient population analysed, arising from four dedicated stroke centres in Europe and analysed by a specialized core laboratory, which gives robustness to our findings.

\section{Conclusion}

The present study highlighted interesting differences between AIS thrombi retrieved from patients treated with bridging-therapy or MT alone. Bridging-therapy clots are smaller and with significantly reduced fibrin and platelet/ other area $\left(\mathrm{mm}^{2}\right)$, but with a percentage of components similar to the MT-alone clots. These findings indicate that IVT administration significantly reduces the size of the thrombus, proportionally releasing all main histological components while carrying out its fibrinolytic activity.

Author contributions KD obtained the funds for the study, developed the study design, coordinated its implementation and supervised the writing of the manuscript; RR participated in samples collection, composed the manuscript, performed the statistical analysis and wrote the results and discussion; SMG performed the ECA measurements, the MSB staining, scanning and quantification; AD, OMM, SF and CT participated in samples collection, the MSB staining, scanning and quantification; PB, SP, AO, GM, GT, AN, AV, KJ, PR, AN, EC, DD, $\mathrm{JC}, \mathrm{KP}, \mathrm{AR}$ and JT performed the thrombectomy at the several hospitals, evaluated the final recanalization outcome and collected samples and procedural data; KP, GT, IS, TT, AR and JT contributed to the study design and were responsible thrombus collection at the relevant stroke center; AP, MG and RM contributed to develop the study design and funding acquisition. All the authors have read and reviewed the manuscript.

Funding Open Access funding provided by the IReL Consortium. This publication has emanated from research conducted with the financial support of Science Foundation Ireland (SFI) and is co-funded under the European Regional Development Fund under Grant Number 13/ RC/2073_2. Furthermore, it was supported by Cerenovus.

Data availability Data may be available upon reasonable request.
Code availability Not applicable.

\section{Declarations}

Conflicts of interest The authors declare no conflict of interest.

Ethical approval This study has been conducted in accordance with the ethical standards of the Declaration of Helsinki and its amendments, by approval of the regional hospital ethics committees and National University of Ireland Galway research ethics committees (16-SEPT-08).

Consent to participate Written informed consent or a waiver of consent was obtained from the patient(s) for their anonymized information to be published in this article.

Consent for publication Not applicable.

Open Access This article is licensed under a Creative Commons Attribution 4.0 International License, which permits use, sharing, adaptation, distribution and reproduction in any medium or format, as long as you give appropriate credit to the original author(s) and the source, provide a link to the Creative Commons licence, and indicate if changes were made. The images or other third party material in this article are included in the article's Creative Commons licence, unless indicated otherwise in a credit line to the material. If material is not included in the article's Creative Commons licence and your intended use is not permitted by statutory regulation or exceeds the permitted use, you will need to obtain permission directly from the copyright holder. To view a copy of this licence, visit http://creativecommons.org/licenses/by/4.0/.

\section{References}

1. Berge E, Whiteley W, Audebert $\mathrm{H}$ et al (2021) European stroke organisation (eso) guidelines on intravenous thrombolysis for acute ischaemic stroke. Eur Stroke J 6:I-LXII

2. Powers William J, Rabinstein Alejandro A et al (2019) Guidelines for the early management of patients with acute ischemic stroke: 2019 update to the 2018 guidelines for the early management of acute ischemic stroke: a guideline for healthcare professionals from the American heart association/American stroke association. Stroke 50:e344-e418

3. Turc G, Bhogal P, Fischer U et al (2019) European stroke organisation (eso)-European society for minimally invasive neurological therapy (esmint) guidelines on mechanical thrombectomy in acute ischemic stroke. J Neurointerv Surg. https://doi.org/10.1136/neuri ntsurg-2018-014569

4. Broderick Joseph P, Hill Michael D (2021) Advances in acute stroke treatment 2020. Stroke 52:729-734

5. Berkhemer OA, Fransen PS, Beumer D, MRCLEAN Investigators et al (2015) A randomized trial of intra-arterial treatment for acute ischemic stroke. N Engl J Med 372:11-20

6. Campbell BC, Mitchell PJ, Kleinig TJ, EXTEND-IA Investigators et al (2015) Endovascular therapy for ischemic stroke with perfusion-imaging selection. N Engl J Med 372:1009-1018

7. Goyal M, Demchuk AM, Menon BK, ESCAPE Trial Investigators et al (2015) Randomized assessment of rapid endovascular treatment of ischemic stroke. N Engl J Med 372:1019-1030

8. Saver JL, Goyal M, Bonafe A, SWIFT PRIME Investigators et al (2015) Stent-retriever thrombectomy after intravenous t-PA vs. t-PA alone in stroke. N Engl J Med 372:2285-2295 
9. Jovin TG, Chamorro A, Cobo E, REVASCAT Trial Investigators et al (2015) Thrombectomy within 8 hours after symptom onset in ischemic stroke. N Engl J Med 372:2296-2306

10. Bracard S, Ducrocq X, Mas JL, THRACE Investigators et al (2016) Mechanical thrombectomy after intravenous alteplase versus alteplase alone after stroke (THRACE): a randomised controlled trial. Lancet Neurol 15:1138-1147

11. Katsanos AH, Malhotra K, Goyal N et al (2019) Intravenous thrombolysis prior to mechanical thrombectomy in large vessel occlusions. Ann Neurol 86(3):395-406

12. Fischer U, Kaesmacher J, Molina CA, Selim MH, Alexandrov AV, Tsivgoulis G (2018) Primary thrombectomy in tPA (tissuetype plasminogen activator) eligible stroke patients with proximal intracranial occlusions. Stroke 49(1):265-326

13. Abilleira S, Ribera A, Cardona P et al (2017) Outcomes after direct thrombectomy or combined intravenous and endovascular treatment are not different. Stroke 48:375-378

14. Rai AT, Boo $S$, Buseman $C$ et al (2018) Intravenous thrombolysis before endovascular therapy for large vessel strokes can lead to significantly higher hospital costs without improving outcomes. J Neurointerv Surg 10:17-21

15. Goyal N, Tsivgoulis G, Pandhi A, Malhotra K, Krishnan R, Ishfaq MF, Krishnaiah B, Nickele C, Inoa-Acosta V, Katsanos AH, Hoit D, Elijovich L, Alexandrov A, Arthur AS (2019) Impact of pretreatment with intravenous thrombolysis on reperfusion status in acute strokes treated with mechanical thrombectomy. J Neurointerv Surg 11(11):1073-1079

16. Rossi R, Fitzgerald S, Molina $S$ et al (2021) The administration of rtPA before mechanical thrombectomy in acute ischemic stroke patients is associated with a significant reduction of the retrieved clot area but it does not influence revascularization outcome. J Thromb Thrombolysis 51(2):545-551. https://doi.org/10.1007/ s11239-020-02279-1

17. Fitzgerald S, Rossi R, Mereuta OM et al (2020) Per-pass analysis of acute ischemic stroke clots: impact of stroke etiology on extracted clot area and histological composition. J Neurointerv Surg. https://doi.org/10.1136/neurintsurg-2020-016966

18. Douglas A, Fitzgerald S, Mereuta OM et al (2020) Platelet-rich emboli are associated with von Willebrand factor levels and have poorer revascularization outcomes. J NeuroInterv Surg 12:557-562

19. De Meyer SF, Andersson T, Baxter B et al (2017) Analyses of thrombi in acute ischemic stroke: a consensus statement on current knowledge and future directions. Int J Stroke 12(6):606-614. https://doi.org/10.1177/1747493017709671

20. Jolugbo P, Ariëns RAS (2021) Thrombus composition and efficacy of thrombolysis and thrombectomy in acute ischemic stroke. Stroke 52(3):1131-1142. https://doi.org/10.1161/STROKEAHA. 120.032810

21. WMA (2013) World medical association Declaration of Helsinki: ethical principles for medical research involving human subjects. JAMA 310(20):2191-2194

22. Goyal M, Fargen KM, Turk AS et al (2014) $2 \mathrm{C}$ or not 2C: defining an improved revascularization grading scale and the need for standardization of angiography outcomes in stroke trials. J Neurointerv Surg 6(2):83-86

23. Adams HP, Bendixen BH, Kappelle LJ et al (1993) Classification of subtype of acute ischemic stroke. Definitions for use in a multicenter clinical trial. TOAST. Trial of Org 10172 in acute stroke treatment. Stroke 24:35-41

24. Fitzgerald S, Rossi R, Mereuta OM et al (2021) Large artery atherosclerotic clots are larger than clots of other stroke etiologies and have poorer recanalization rates. J Stroke Cerebrovasc Dis 30(1):105463. https://doi.org/10.1016/j.jstrokecerebrovasdis. 2020.105463

25. Rossi R, Fitzgerald S, Mereuta OM et al (2021) Correlation between acute ischaemic stroke clot length before mechanical thrombectomy and extracted clot area: impact of thrombus size on number of passes for clot removal and final recanalization. Eur Stroke J. https://doi.org/10.1177/23969873211024777

26. Fitzgerald ST, Wang S, Dai D et al (2019) Platelet-rich clots as identified by Martius scarlet blue staining are isodense on NCCT. J Neurointerv Surg 11:1145-1149

27. Fitzgerald S, Wang S, Dai D et al (2019) Orbit image analysis machine learning software can be used for the histological quantification of acute ischemic stroke blood clots. PLoS ONE 14:e0225841

28. Stritt M, Stalder AK, Vezzali E (2020) Orbit image analysis: An open-source whole slide image analysis tool. PLoS Comput Biol 16:e1007313

29. Meyer BC, Lyden PD, Faha F (2009) The modified national institutes of health stroke scale (mNIHSS): its time has come. Int J Stroke 4(4):267-273

30. Krajičcková D, Krajina A, Šteiner I et al (2018) Fibrin clot architecture in acute ischemic stroke treated with mechanical thrombectomy with stent-retrievers-cohort study. Circ J 82:866-873

31. Stanford SN, Sabra A, D'Silva L et al (2015) The changes in clot microstructure in patients with ischaemic stroke and the effects of therapeutic intervention: a prospective observational study. BMC Neurol 15:35

32. Mereuta OM, Fitzgerald S, Christensen TA, Jaspersen AL, Dai D, Abbasi M, Puttappa T, Kadirvel R, Kallmes DF, Doyle KM, Brinjikji W (2020) High-resolution scanning electron microscopy for the analysis of three-dimensional ultrastructure of clots in acute ischemic stroke. J Neurointerv Surg. https://doi.org/10.1136/neuri ntsurg-2020-016709

33. Choi MH, Park GH, Lee JS, Lee SE, Lee SJ, Kim JH, Hong JM (2018) Erythrocyte fraction within retrieved thrombi contributes to thrombolytic response in acute ischemic stroke. Stroke 49:652-659

34. Kim YD, Nam HS, Kim SH et al (2015) Time-dependent thrombus resolution after tissue-type plasminogen activator in patients with stroke and mice. Stroke 46:1877-1882

35. Gersh KC, Nagaswami C, Weisel JW (2009) Fibrin network structure and clot mechanical properties are altered by incorporation of erythrocytes. Thromb Haemost 102:1169-1175

36. Carr ME Jr, Hardin CL (1987) Fibrin has larger pores when formed in the presence of erythrocytes. Am J Physiol 253(5 Pt 2):H1069-H1073 


\section{Authors and Affiliations}

Rosanna Rossi ${ }^{1,2} \cdot$ Sara Molina ${ }^{1,2} \cdot$ Oana Madalina Mereuta ${ }^{1,2} \cdot$ Andrew Douglas $^{1,2} \cdot$ Seán Fitzgerald ${ }^{1}$. Ciara Tierney ${ }^{1,2}$ - Abhay Pandit ${ }^{2}$. Paul Brennan ${ }^{3}$ - Sarah Power ${ }^{3}$ - Alan O'Hare ${ }^{3}$ - Michael Gilvarry ${ }^{4}$. Ray McCarthy ${ }^{4}$. Georgios Magoufis ${ }^{5} \cdot$ Georgios Tsivgoulis $^{6} \cdot$ András Nagy $^{7}$. Ágnes Vadász ${ }^{7} \cdot$ Katarina Jood $^{8,10}$. Petra Redfors ${ }^{8,10}$. Annika Nordanstig $^{8,10}$. Erik Ceder $^{9} \cdot$ Dennis Dunker $^{9} \cdot$ Jeanette Carlqvist $^{9} \cdot$ Klearchos Psychogios $^{5} \cdot$ István Szikora $^{7}$. Turgut Tatlisumak $^{8,10} \cdot$ Alexandros Rentzos $^{9} \cdot$ John Thornton $^{3} \cdot$ Karen M. Doyle ${ }^{1,2}$ (1)

1 Department of Physiology and Galway Neuroscience Centre, School of Medicine, National University of Ireland Galway, University Road, Galway, Ireland

2 CÚRAM-SFI Research Centre in Medical Devices, National University of Ireland Galway, Galway, Ireland

3 Department of Radiology, Royal College of Surgeons in Ireland, Beaumont Hospital, Dublin, Ireland

4 Cerenovus, Galway, Ireland

5 Metropolitan Hospital, Stroke Unit, Piraeus, Greece

6 Second Department of Neurology, National and Kapodistrian University of Athens, "Attikon" University Hospital, Athens, Greece
7 Department of Neurointerventions, National Institute of Clinical Neurosciences, Budapest, Hungary

8 Department of Neurology, Sahlgrenska University Hospital, Gothenburg, Sweden

9 Department of Interventional and Diagnostic Neuroradiology, Sahlgrenska University Hospital, University of Gothenburg, Gothenburg, Sweden

10 Department of Clinical Neuroscience, Institute of Neuroscience and Physiology, Sahlgrenska Academy at University of Gothenburg, Gothenburg, Sweden 